\title{
Is fresh frozen plasma effective for thrombocytopenia in adults with dengue fever? A prospective randomised double blind controlled study
}

\author{
K H Sellahewa ${ }^{1}$, N Samaraweera' ${ }^{1}$ K P G D Thusita ${ }^{1}$ and J L I N Fernando ${ }^{1}$ \\ (Index words: dengue fever, thrombocytopenia, fresh frozen plasma)
}

\begin{abstract}
Rationale Thrombocytopenia is a common problem which causes concern and complications in dengue fever. If proven effective, intravenous fresh frozen plasma is a simple and widely available therapeutic option to manage thrombocytopenia.

Objective To test the efficacy of fresh frozen plasma (FFP) on thrombocytopenia in patients with dengue fever.

Design 109 serologically confirmed dengue patients with platelet counts $<40000 / \mathrm{mm}^{3}$ were randomised into two groups. Group A (treatment) comprised 53 patients and group $B$ (control) 56 patients. Group A received an intravenous infusion of 3 units $(600 \mathrm{ml})$ of FFP over 90 minutes. Group B received an intravenous infusion of an equal volume of isotonic saline over the same period. The primary outcome measure was the difference between pre- and post-interventional platelet counts at 12, 24 and 48 hours.
\end{abstract}

Results Following Intervention, the mean platelet count was significantly higher in Group A than in Group B at 12 hours ( $p=0.04$; t-test). The mean platelet counts continued to be higher in Group A than in Group B at 24 and 48 hours post-intervention, but the differences were not statistically significant.

Conclusions In dengue patients with thrombocytopenia, infusion of $600 \mathrm{ml}$ FFP may contribute to a significant increase in platelet count in the first 12 hours, but not thereafter.

\section{Introduction}

Thrombocytopenia is a common problem in dengue which causes concern to both patients and doctors. There are no clear guidelines for its management. Irrational and inappropriate interventions and transfer of patients from peripheral hospitals to tertiary care hospitals, primarily for platelet transfusions, reflect the dilemmas in managing this complication.

The pathogenesis of thrombocytopenia in dengue fever is not clearly understood. Increased peripheral destruction of antibody coated platelets is implicated [1, $2]$. Other mechanisms described are bone marrow hypocellularity due to destructive effects of the virus on bone marrow [3] and platelet destruction in the liver and spleen. It is possible that antibody concentrates in the form of FFP could block immune mediated platelet destruction. The postulated mechanisms are akin to $\mathrm{Fc}$ receptor blockade by intravenous immunoglobulin and the associated inhibition of platelet binding by macrophages. This could lead to a reduction in peripheral platelet destruction, and hence an increase in the platelet count. In addition, thrombopoeitin activator in FFP may also directly stimulate thrombopoeitin in the bone marrow $[4,5]$.

The tendency of most clinicians is to transfuse platelet rich plasma (PRP) when managing thrombocytopenia [6]. However, platelets present a strong antigenic stimulus, which by evoking an exalted immune response, could cause further immune mediated platelet destruction. Consequently, platelet counts of dengue patients fluctuate in an unpredictable manner despite platelet transfusion. Platelet rich plasma is costly, needs specialised skills and machinery, and can be stored for only a few hours. FFP is easier to prepare, could be stored for 6 months, and is freely available even in peripheral settings.

\section{Objective}

The objective of this study was to test the effects of FFP on thrombocytopenia in patients with dengue fever.

\section{Methods}

\section{The null hypothesis}

Our null hypothesis was 'there is no improvement of platelet counts of thrombocytopenic dengue patients following treatment with intravenous FFP'.

\section{Study design}

The study was done from November 2005 to August 2006. The study population comprised patients with clinical evidence and serological confirmation of dengue

${ }^{1}$ National Hospital of Sri Lanka, Colombo, Sri Lanka.

Correspondence: KHS, e-mail <kolithah@gmail.com>. Competing Interests: none declared. Received 7 September 2007 and revised version accepted 9 February 2008. Clinical Trials Registry No: SLCTR/2007/004. 
fever admitted to one medical unit at the National Hospital of Sri Lanka, Colombo.

Clinical criteria for diagnosis were based on features of an acute viral fever during a dengue epidemic, namely, fever with chills, myalgia, arthralgia, headache, and diffuse blanching skin erythema [7]. Confirmatory serological tests were either dengue specific IgM determined by enzyme-linked immunosorbent assay (Panbio, Australia) or a haemagglutination inhibition titre of $\geq 1: 2560$ [8]. Patients who were included in the study had a platelet count below $40000 / \mathrm{mm}^{3}$. Patients with a platelet count below $10000 / \mathrm{mm}^{3}$, dengue shock syndrome, or who were considered to be seriously ill by the attending physician were excluded.

Sample size was calculated using the estimated standard deviation of 7.7 which was derived from a previous study [9] and a difference of 5 points was considered to be of clinical importance. We calculated that a sample size of 76 was needed to give the study $80 \%$ power at $\alpha=0.05$.

Patients were randomised using a random-numbers table into treatment (Group A) and control (Group B) groups using sealed envelopes by a person who was not involved in the study. 109 patients were randomised. Group A (53 patients) received an intravenous infusion of 3 units ( $600 \mathrm{ml})$ of FFP over 90 minutes. Group B (56 patients) received an intravenous infusion of an equal volume of isotonic saline over the same period. In both groups platelet counts were done at 12, 24 and 48 hours after the infusion. All the platelet counts were done in the same laboratory using the same machine to ensure reliability and repeatability. No other intravenous fluids were given during this time. All patients received paracetamol $1 \mathrm{~g} 6$ hourly as an antipyretic analgesic, but no other drugs. The study protocol permitted the use of inotropes or other supportive care as appropriate. Clinical assessment was done by a physician who remained unaware of the treatment assignments throughout the study. The assessment involved detection of any side-effects, such as anaphylactic reactions, which were recorded. Discharge of patients from hospital was decided by the same physician.

Approval for the study was obtained from the Scientific and Ethical Review Committee of the Faculty of Medicine, University of Colombo, as well as the Ethical Review Board of the National Hospital of Sri Lanka. All patients gave written informed consent.

\section{Outcome measures}

The primary outcome measures were the platelet counts at 12, 24 and 48 hours, post-intervention.

\section{Data collection and analysis}

Individual patient data were collected by a trained observer (blind to treatment) on a pre-designed data sheet according to the allocated serial number. Information regarding the patient's age, sex, BMI, mode of admission, presence or absence of bleeding manifestations, lymphadenopathy, pre- and post-intervention blood pressure, complications before or during the hospital stay, and dengue serology were recorded. The core data consisted of platelet counts, $\mathrm{PCV}, \mathrm{Hb}$ and $\mathrm{WBC}$ before and at 12, 24, and 48 hours after intervention.

\section{Statistical analysis}

Data were entered into a database created on Microsoft Excel. Data analysis was carried out by Statistical Package for Social Science (SPSS) version 12.0 (SPSS inc, Chicago III). Two independent sample t-tests were used to compare mean platelet counts between the two groups at baseline and at 12, 24 and 48 hours after intervention, and within each group at 12,24 and 48 hours against the pre-intervention value. Chi-square test was used to compare categorical variables. Statistical results were re-tested by EPI Info version 6.01b. A p-value of less than 0.05 was accepted as evidence of statistical significance. We analysed data on an intention to treat basis, blind to the allocated treatment.

\section{Results}

The two groups were matched for baseline characteristics such as age, sex ratio, BMI, clinical features, and pre-intervention platelet counts (table 1). Figure 1 shows the study algorithm. One patient in Group A developed an allergic reaction to FFP during the study, and could not be given the full dose. All 53 patients in Group A were included in the final analysis. One patient in Group B was excluded as he was also diagnosed as having idiopathic thrombocytopenic purpura. 3 patients developed shock during the study, and were treated accordingly. Data from these patients were analysed on an intention to treat basis. Fifty five patients in Group B were included in the final analysis. There were no deaths among the patients enrolled in the study.

Table 2 shows the mean platelet counts of dengue patients in group A and group B during the period of study. Pre-intervention mean platelet counts were similar in the two groups. Following intervention, the mean platelet count was significantly higher in Group A than in Group B at 12 hours ( $p=0.04$; t-test). The mean platelet counts continued to be higher in Group A than in Group B at 24 and 48 hours post-intervention, but the differences were not statistically significant. The percentage of patients who had a fall in their platelet counts at 12, 24 and 48 hours post-intervention were $13.2 \%, 5.7 \%, 1.9 \%$ respectively in Group A and 20\%, 12.7\% and 5.5\% respectively in Group B $(2=0.38, p=0.54$, Chi-square for linear trend). 


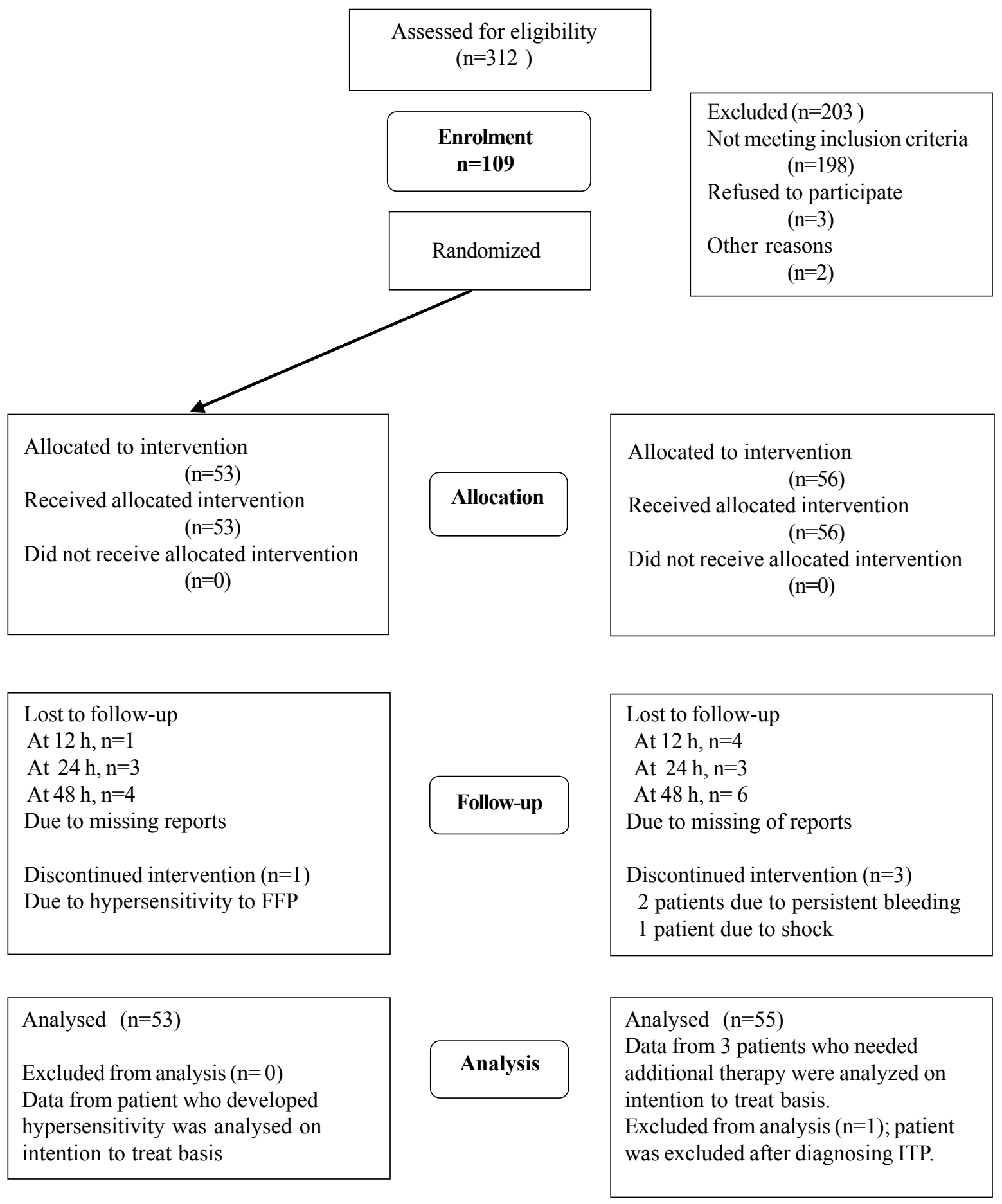

Figure 1. Study algorithm 
Table 1. Baseline characteristics of the study groups

\begin{tabular}{|c|c|c|c|}
\hline \multirow[t]{2}{*}{ Characteristics } & \multicolumn{2}{|c|}{ Study groups } & \multirow[t]{2}{*}{ Significance } \\
\hline & Group $A(n=53)$ & Group B $(n=55)$ & \\
\hline \multicolumn{4}{|l|}{ Age (years) } \\
\hline Mean \pm SD & $28.8 \pm 11.8$ & $30.0 \pm 11.1$ & $\mathrm{p}=0.69^{*}$ \\
\hline \multicolumn{4}{|l|}{ Sex } \\
\hline Male : female & $42: 11$ & $39: 16$ & $\mathrm{p}=0.37 * *$ \\
\hline \multicolumn{4}{|l|}{ BMI } \\
\hline Mean \pm SD & $22.3 \pm 2.6$ & $22.6 \pm 2.4$ & $\mathrm{p}=0.57^{*}$ \\
\hline \multicolumn{4}{|c|}{ Cervical lymph nodes } \\
\hline Positive & $16(30.2 \%)$ & $19(34.5 \%)$ & \\
\hline Negative & $37(69.8 \%)$ & $36(65.5 \%)$ & $\mathrm{p}=0.64 * *$ \\
\hline \multicolumn{4}{|c|}{ Bleeding manifestations } \\
\hline Positive & $14(26.4 \%)$ & $15(27.3 \%)$ & \\
\hline Negative & $39(73.6 \%)$ & $40(72.7 \%)$ & $\mathrm{p}=0.92 * *$ \\
\hline \multicolumn{4}{|l|}{ Fever } \\
\hline Positive & 53 & 55 & \\
\hline \multicolumn{4}{|c|}{ Diffuse blanching erythema } \\
\hline Positive & $47(88.7 \%)$ & $51(92.7 \%)$ & \\
\hline Negative & $6(11.3 \%)$ & $4(7.3 \%)$ & $\mathrm{P}=0.31 * *$ \\
\hline
\end{tabular}

$*$ t-test, $* *$ chi-square test

Table 2. Comparison of platelet counts (in thousands) after intervention

\begin{tabular}{llllll}
\hline Observed period & \multicolumn{3}{c}{ Study group } & & $\begin{array}{c}\text { Significance } \\
(\text { t-test, } p)\end{array}$ \\
\cline { 2 - 5 } & \multicolumn{2}{c}{ Group A } & Mean $(S D)$ & $n$ & \\
\cline { 2 - 5 } & Mean $(S D)$ & $n$ & $33.1(11.0)$ & 55 & $\mathrm{t}=0.85, \mathrm{p}=0.39$ \\
Pre-intervention & $31.2(11.3)$ & 53 & $18.4(26.5)$ & 51 & $\mathrm{t}=2.05, \mathrm{p}=0.04$ \\
After 12 h & $31.4(37.1)$ & 52 & $40.2(30.1)$ & 52 & $\mathrm{t}=1.14, \mathrm{p}=0.26$ \\
After 24 h & $49.7(44.7)$ & 50 & $84.7(62.8)$ & 49 & $\mathrm{t}=1.58, \mathrm{p}=0.12$ \\
After 48 h & $105.1(64.9)$ & 49 & & &
\end{tabular}




\section{Comment}

Our results indicate that in dengue patients with thrombocytopenia infusion of $600 \mathrm{ml}$ FFP may contribute to an increase in platelet count in the first 12 hours, but not thereafter. The kinetics of platelet counts in dengue is that counts rise after an initial decrease [9]. The absence of a significant difference in platelet counts between the two groups at 24 and 48 hours post-intervention probably reflects this natural history. Thus, FFP may be useful in dengue patients with thrombocytopenia only early in the disease, when platelets counts are decreasing. As the association between severe thrombocytopenia and severe dengue is well recognised, patients with severe thrombocytopenia early in the disease, with other variables which predict progression to severe disease, such as, a low pulse pressure, poor capillary filling, and evidence of plasma leakage, may benefit from FFP infusion.

\section{References}

1. Saito M, Oishi K, Inoue S. Association of increased plateletassociated immunoglobulins with thrombocytopenia and the severity of disease in secondary dengue virus infections. Clinical and Experimental Immunology 2004; 138: 299-303.

2. Nakao S, Lai CJ, Young NS. Dengue virus, a flavivirus, propagates in human bone marrow progenitors and hematopoietic cell lines. Blood 1989; 74: 1235-40.
3. Murgue B, Cassar O, Guigon M, Chungue E. Dengue virus inhibits human hematopoietic progenitor growth in vitro. Journal of Infection Disease 1997; 175: 1497-501.

4. De Sauvage FJ, Carver-Moore K, Luoh SM, et al. Physiological regulation of early and late stages of megakaryocytopoiesis by thrombopoietin. Journal of Experimental Medicine 1996; 183: 651-6.

5. Li J, Xia Y, Kuter DJ. Interaction of thrombopoietin with the platelet c-mpl receptor in plasma: binding, internalization, stability and pharmacokinetics. British Journal of Haematology 1999; 106: 345-56.

6. Kumar ND, Tomar V, Singh B, Kela K. Platelet transfusion practice during dengue fever epidemic. Indian Journal of Pathology and Microbiology 2000; 43: 55-60.

7. Kularatne SAM, Gawarammana IB, Kumarasiri PRV. Epidemiology, clinical features, laboratory investigations and early diagnosis of dengue fever in adults: a descriptive study in Sri Lanka. Southeast Asian Journal of Tropical Medicine 2005; 36: 686-92.

8. Videa E, Coloma MJ. Immunoglobulin M enzyme-linked immunosorbent assay using recombinant polypeptides for diagnosis of dengue. Clinical Diagnostic Laboratory Immunology 2005; 12: 882-4.

9. Taid YH, Chee YC, Chan KW. The natural history of dengue illness based on a study of hospitalised patients in Singapore. Singapore Medical Journal 1999; 40: 238-42. 